\title{
Reliability and validity of CODA motion analysis system for measuring cervical range of motion in patients with cervical spondylosis and anterior cervical fusion
}

\author{
ZHONGYANG GAO ${ }^{1}$, HUI SONG ${ }^{1}$, FENGGANG REN ${ }^{2}$, YUHUAN LI ${ }^{1}$, DONG WANG $^{1}$ and XIJING HE ${ }^{1}$ \\ ${ }^{1}$ Department of Orthopedics, The Second Affiliated Hospital of Xi'an Jiaotong University, Xi'an, Shaanxi 710004; \\ ${ }^{2}$ Department of Surgery, The Research Institute of Advanced Surgical Techniques and \\ Engineering of Xi'an Jiaotong University, Xi'an, Shaanxi 710061, P.R. China
}

Received April 12, 2017; Accepted August 1, 2017

DOI: $10.3892 / \mathrm{etm} .2017 .5239$

\begin{abstract}
The aim of the present study was to evaluate the reliability of the Cartesian Optoelectronic Dynamic Anthropometer (CODA) motion system in measuring the cervical range of motion (ROM) and verify the construct validity of the CODA motion system. A total of 26 patients with cervical spondylosis and 22 patients with anterior cervical fusion were enrolled and the CODA motion analysis system was used to measure the three-dimensional cervical ROM. Intra- and inter-rater reliability was assessed by interclass correlation coefficients (ICCs), standard error of measurement (SEm), Limits of Agreements (LOA) and minimal detectable change (MDC). Independent samples t-tests were performed to examine the differences of cervical ROM between cervical spondylosis and anterior cervical fusion patients. The results revealed that in the cervical spondylosis group, the reliability was almost perfect (intra-rater reliability: ICC, 0.87-0.95; LOA, -12.86-13.70; SEm, 2.97-4.58; inter-rater reliability: ICC, 0.84-0.95; LOA, -13.09-13.48; SEm, 3.13-4.32). In the anterior cervical fusion group, the reliability was high (intra-rater reliability: ICC, 0.88-0.97; LOA, -10.65-11.08; SEm, 2.10-3.77; inter-rater reliability: ICC, 0.86-0.96; LOA, -10.91-13.66; SEm, 2.20-4.45). The cervical ROM in the cervical spondylosis group was significantly higher than that in the anterior cervical fusion group in all directions except for left rotation. In conclusion, the CODA motion analysis system is highly reliable in measuring cervical ROM and the construct validity was verified, as the system was sufficiently
\end{abstract}

Correspondence to: Professor Xijing He, Department of Orthopedics, The Second Affiliated Hospital of Xi'an Jiaotong University, 157 Xiwu Road, Xi'an, Shaanxi 710004, P.R. China E-mail: he_xijing123@126.com

Key words: Cartesian Optoelectronic Dynamic Anthropometer motion system, cervical range of motion, reliability, validity, cervical spondylosis, anterior cervical fusion sensitive to distinguish between the cervical spondylosis and anterior cervical fusion groups based on their ROM.

\section{Introduction}

Cervical spondylosis is the most common spine disorder in the elderly (1). Certain patients require anterior cervical fusion (ACF) surgery for preventing any further impairment of neurological function. Due to neck pain, cervical spine degeneration and cervical fusion, numerous cervical spondylosis and ACF patients suffer from limitations of cervical mobility $(2,3)$ and cannot maintain their life quality; this includes difficulty of walking down stairs, washing their hair and driving cars. As an important indicator of cervical disease and postoperative life quality (4), the cervical range of motion (CROM) is routinely measured by clinicians and researchers to evaluate the cervical impairment, therapeutic effect and residual disability.

However, due to the complex anatomy of the cervical spine and the resulting coupled movement, it is challenging to determine the CROM measurement accurately and reliably (5). At present, clinicians and researchers utilize various devices to assess CROM. When raters use goniometry (6), inclinometry (7), radiography (8) or a CROM device (9) for assessment, no continuous angles are obtained, and scales require to be consulted or complex calculations must be performed, which are sources of human error. Furthermore, the CROM device cannot measure the pure CROM but includes the trunk motion. As to electromagnetic motion analysis (10), ultrasound motion analysis (11) and optical motion analysis (12), they do not achieve 'good' reliability and validity in all directions of CROM. Thus, no optimal device for measuring CROM in clinical and research practice is currently available.

The Cartesian Optoelectronic Dynamic Anthropometer (CODA) motion analysis system (Charnwood Dynamics Ltd., Rothley, UK) is a real-time three-dimensional kinematic analysis instrument. This system has been employed in the analysis of gait posture (13), lower and upper extremity kinematics $(14,15)$ and lumbar spine ROM (16) in various populations and circumstances, and proved to be reliable and accurate. To the best of our knowledge, it has not been applied in the assessment of cervical mobility (17). 
Considering the advantages of directly measuring the CROM as well as the CODA motion system being accurate and reliable in the analysis of other motions, the present study first applied the system for measuring the CROM, evaluated the intra- and inter-rater reliability and verified the construct validity of the system to discriminate between cervical spondylosis and ACF patients (known group construct validity).

\section{Materials and methods}

Subjects. A total of 26 patients with cervical spondylosis and 22 patients with ACF who presented at the Second Affiliated Hospital of Xi'an Jiaotong University (Xi'an, China) between September 2015 and September 2016 were enrolled in the present study. The demographic data of patients was collected, and the visual analogue scale (VAS) (18) and Japanese Orthopaedic Association (JOA) score (19) were recorded. The patients of the cervical spondylosis group were diagnosed with cervical spondylotic radiculopathy or cervical spondylotic myelopathy by clinical systems and magnetic resonance imaging examination. The patients of the ACF group had received single- or multi-level anterior cervical discectomy and fusion (ACDF) or anterior cervical corpectomy and fusion (ACCF) due to cervical spondylosis, and solid cervical fusion was confirmed by X-ray and computed tomography scan. Informed consent forms were signed by all participants prior to measurements and ethical approval was provided by the Ethical Committee of Xi'an Jiaotong University (Xi'an, China).

Instruments. The CODA motion analysis system was used to collect the cervical kinematic data. The system is composed of marker devices, sensor modules and data analysis software. The marker devices consist of infrared emission markers and drive boxes, which are attached to the skin. The sensor modules are made up of three optical sensors, which capture the vertical, horizontal and rotational movements by tracking the markers' position in real-time. The data analysis software, CODA motion ODIN, analyzes the data from the sensor modules and records the three-dimensional CROM. During the measurements of CROM, two sensor modules were set-up at two diagonal sides of the test room, and three drive boxes and seven markers were selected to capture the head, cervical and thoracic movements. Marker 0 and marker 1 of drive box A were attached to glabella and mental protuberance, respectively. Markers 0,1 and 2 of drive box B were attached to the external occipital protuberance, the seventh cervical vertebra (C7) and the eighth thoracic vertebra, respectively. Markers 0 and 1 of drive box $C$ were attached to the incisure jugular and processus xiphoideus, respectively (Fig. 1).

Procedures. A low-back chair was placed between two sensor modules and the patients were requested to sit up facing one sensor module. Their arms rested on the knees and their feet were kept flat on the ground. The patients were required to wear swimming caps to prevent interference of head hair. Prior to the measurements, all patients performed three repetitions of cervical motion in each direction as a warm-up. During the measurements, the patients were required to move in the following order: Flexion, extension, right lateral bending, left lateral bending, right axial rotation and left axial rotation.
They were also asked to move their heads as much as possible in each direction without any discomfort of the cervical spine, while the shoulders and trunk were kept in a static position. At the end of each movement, the patients remained static for $1 \mathrm{sec}$ in case the sensor module missed the signal, and then slowly returned to the neutral position.

Two orthopedics postgraduate students of Xi'an Jiaotong University were selected as the testers (testers A and B). They were provided with specific training of operating the CODA motion analysis system prior to the study. The CROM of each patient was measured with three repetitions, two of which were performed by tester $\mathrm{A}(\mathrm{A} 1, \mathrm{~A} 2)$ and the other one by tester $\mathrm{B}$ (B1). There was a 1-week interval between $\mathrm{A} 1$ and $\mathrm{A} 2$, and $\mathrm{A} 2$ was be performed at the same time of day as A1 to reduce the influence of diurnal variation. B1 was performed on the same day as A1 with a 20 -min interval between the two testers, and tester $\mathrm{B}$ was blinded to the previous measurements. The intra-rater reliability was evaluated from the results of A1 and $\mathrm{A} 2$, and inter-rater reliability was evaluated from the results of $\mathrm{A} 1$ and $\mathrm{B} 1$.

Statistical analysis. All statistical analyses were performed by using SPSS 19.0 software (IBM Corp., Armonk, NY, USA). All data were proved to be normally distributed by the Kolmogorov-Smirnov test and thus, to assess the differences between groups, independent samples t-tests were performed. The characteristics of patients were described as the mean \pm standard deviation (SD).

The reliability of the measurements in cervical spondylosis and ACF patients was calculated. Intra- and inter-rater reliability was assessed by determining the intraclass correlation coefficient (ICC) with 95\% confidence intervals $(95 \% \mathrm{CI})$. The one-way random model was used to calculate the ICC for intra-rater reliability, and the two-way random model was used for inter-rater reliability. Standard error of measurement (SEm) was calculated as SDx $\sqrt{1}$-ICC, where SD refers to the SD of the combined values of two tests (20). The minimal detectable change (MDC) was calculated for a 95\% confidence level, and the formula is MDC $=1.96 \times \sqrt{ } 2 \mathrm{SEm}(21)$. Limits of agreements (LOA) were calculated as the mean difference $\pm 1.96 \mathrm{SD}$ of the differences (22). Bland-Altman plots were also generated to display the graphical differences between the measurements of two tests (22). According to the categorization of Shrout (23), an ICC value of $>0.8$ was considered as highly reliable.

To verify the known group construct validity (24), an independent samples t-test was performed to examine the differences in CROM between cervical spondylosis and ACF patients, and the value of CROM in each direction was calculated as the average value of $\mathrm{A} 1, \mathrm{~A} 2$ and $\mathrm{B} 1 . \mathrm{P}<0.05$ was considered to indicate a statistically significant difference.

\section{Results}

Subjects. The characteristics of the patients in the cervical spondylosis and ACF groups are displayed in Table I. The cervical spondylosis group was composed of 26 subjects, and the clinical diagnoses of patients included myelopathy, radiculopathy or both. The ACF group was composed of 22 subjects and the surgical options included one- or two-level ACDF and 
Table I. Characteristics of patients.

\begin{tabular}{lccr}
\hline Parameter & Cervical spondylosis $(\mathrm{n}=26)$ & Anterior cervical fusion $(\mathrm{n}=22)$ & P-value \\
\hline Sex (female/male) & $12 / 14$ & $13 / 9$ & 0.37 \\
Age (years) & $55.8 \pm 12.5$ & $56.8 \pm 11.3$ & 0.26 \\
Diagnosis & & & \\
Myelopathy & 13 & N/A & N/A \\
Radiculopathy & 8 & N/A & N/A \\
Mixed & 4 & N/A & N/A \\
Surgical option & & & N/A \\
One-level ACDF & N/A & 4 & N/A \\
Two-level ACDF & N/A & 6 & N/A \\
One-level ACCF & N/A & 6 & N/A \\
Two-level ACCF & N/A & 6 & $<0.001$ \\
VAS score & $6.3 \pm 1.3$ & $2.3 \pm 0.8$ & $<0.001$ \\
JOA score & $10.3 \pm 1.7$ & $14.2 \pm 1.4$ & \\
\hline
\end{tabular}

Values are expressed as the mean \pm standard deviation or $\mathrm{n}$. ACDF, anterior cervical discectomy and fusion; ACCF, anterior cervical corpectomy and fusion; VAS, visual analogue scale; JOA, Japanese Orthopaedic Association; N/A, not available.

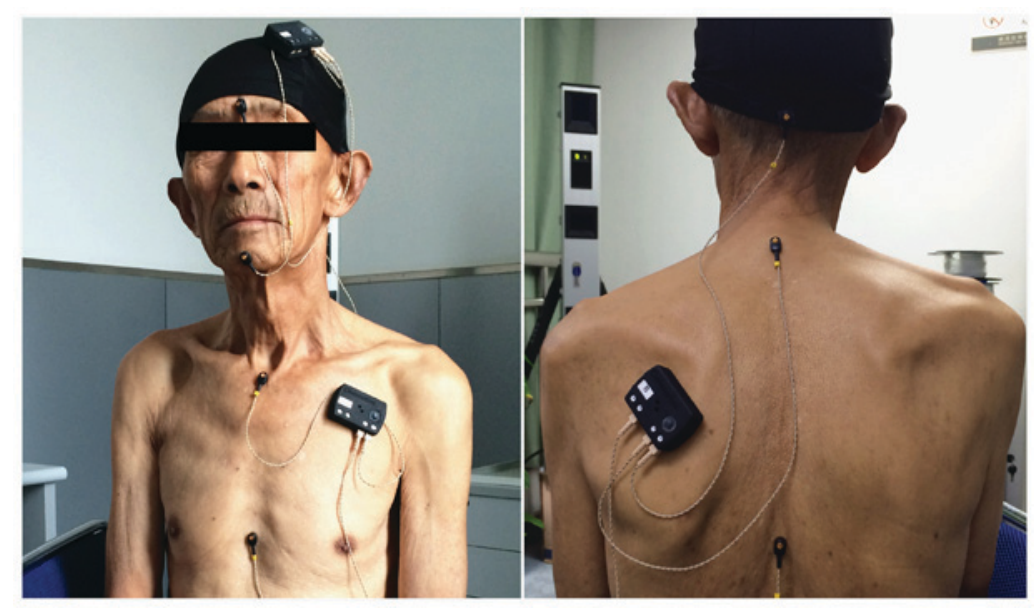

Figure 1. Position of the markers on the head and trunk: The position of markers included the glabella, mental protuberance, external occipital protuberance, the seventh cervical vertebra, the eighth thoracic vertebra, incisure jugular and processus xiphoideus.

one- or two-level ACCF. The mean follow-up time in the ACF group was $6.7 \pm 2.1$ months. The differences in sex distribution and age between the groups were not significant. However, significant differences in VAS score and JOA score were identified between the two groups.

Reliability study on the cervical spondylosis group. The mean CROM as well as the ICC (95\% CI), LOA, SEm and MDC according to the intra- and inter-rater reliability study are displayed in Table II. The ROM in lateral bending ranked lowest, following the extension/flexion, and the axial rotation ranked highest according to the intra- as well as the inter-rater analysis. The ICC values according to the intra-rater reliability analysis, ranging from 0.87 (flexion) to 0.95 (right axial rotation), were slightly better than those according to the inter-rater reliability analysis, which ranged from 0.84 (flexion) to 0.95 (right axial rotation), indicating a high level of reliability. The LOA ranged from -12.86 to 13.70 and -13.09 to 13.48 according to the intra- and inter-rater analysis, respectively. The SEm according to the intra- and inter-rater reliability analysis was similar, ranging from 2.97 to 4.58 and 3.13 to 4.32 , respectively. The MDC ranged from 8.23 to 12.69 according to the intra-rater analysis and from 8.67 to 11.97 according to the inter-rater analysis. Figs. 2 and 3 display the intra- and inter-rater LOA plots for total CROM in the cervical spondylosis group, respectively.

Reliability study on the ACF group. The mean CROM as well as the ICC $(95 \% \mathrm{CI}), \mathrm{LOA}, \mathrm{SEm}$ and MDC according to the intra- and inter-rater reliability analysis are displayed in Table III. The highest ROM was in the axial rotation direction and the lowest ROM was in the lateral bending direction according to the intra- as well as the inter-rater analysis. The ICC values ranged from 0.88 (extension) to 0.97 (right axial 


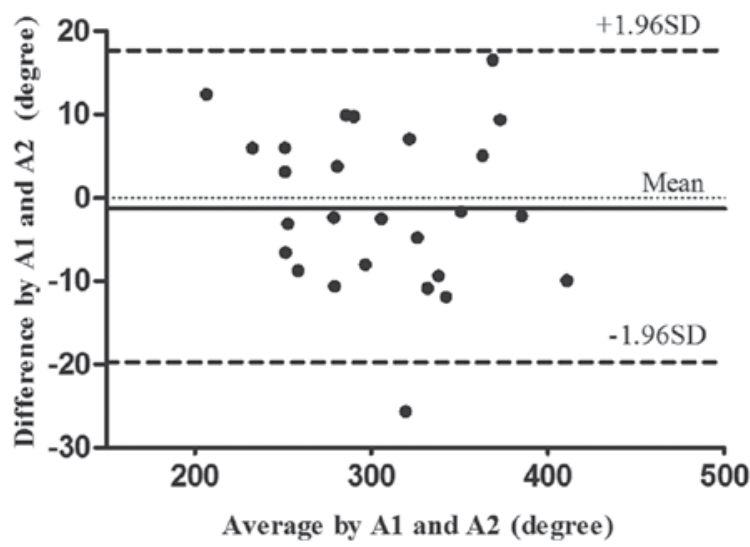

Figure 2. Intra-rater limits of agreements plots for total cervical range of motion of the cervical spondylosis group. The solid line indicates the mean difference of the measurements, the upper and lower dashed lines indicate the $1.96 \mathrm{SD}$ above and below the mean difference of the measurements. SD, standard deviation; A1, first measurement by tester A; A2, second measurement by tester A (1 week after A1).

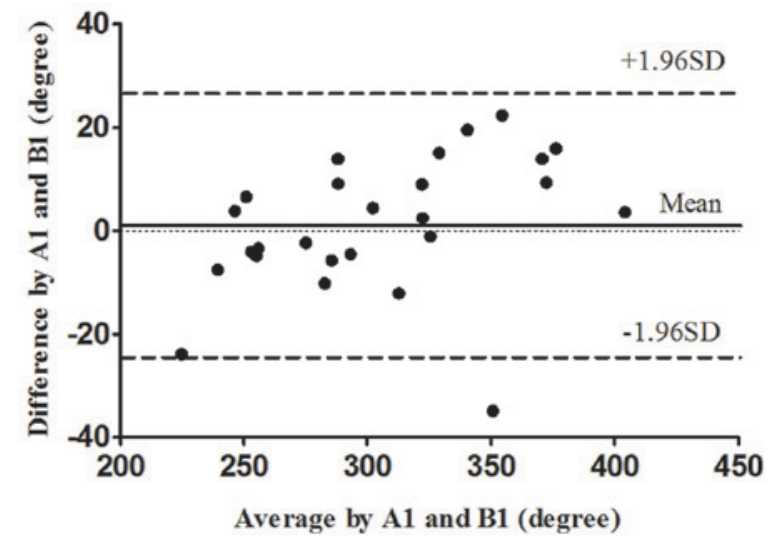

Figure 3. Inter-rater limits of agreements plots for total cervical range of motion of the cervical spondylosis group. The solid line indicates the mean difference of the measurements, the upper and lower dashed lines indicate the 1.96 SD above and below the mean difference of the measurements. SD, standard deviation; A1, measurement by tester A; B1, measurement by tester B ( 20 min after A1).

rotation) according to the intra-rater analysis and 0.86 (extension) to 0.96 (right lateral bending) according to the inter-rater analysis, revealing almost perfect reliability. According to the intra-rater analysis, the LOA ranged from -10.65 to 11.08 , the SEm ranged from 2.10 to 3.77 and the MDC ranged from 5.82 to 10.44. Similar to the inter-rater analysis results, the LOA ranged from -10.91 to 13.66, the SEm ranged from 2.20 to 4.45 and the MDC ranged from 6.09 to 12.33 . Figs. 4 and 5 display the intra- and inter-rater LOA plots for the total CROM in the ACF group, respectively.

Construct validity. The inter-group differences in CROM are displayed in Table IV. The CROM regarding flexion, extension, right lateral bending, left lateral bending and right axial rotation in the ACF group was significantly lower than that in the cervical spondylosis group $(\mathrm{P} \leq 0.041)$, and a trend towards a lower ROM in left axial rotation was also noted in the ACF group, but the difference between groups was not significant $(\mathrm{P}=0.120)$. 


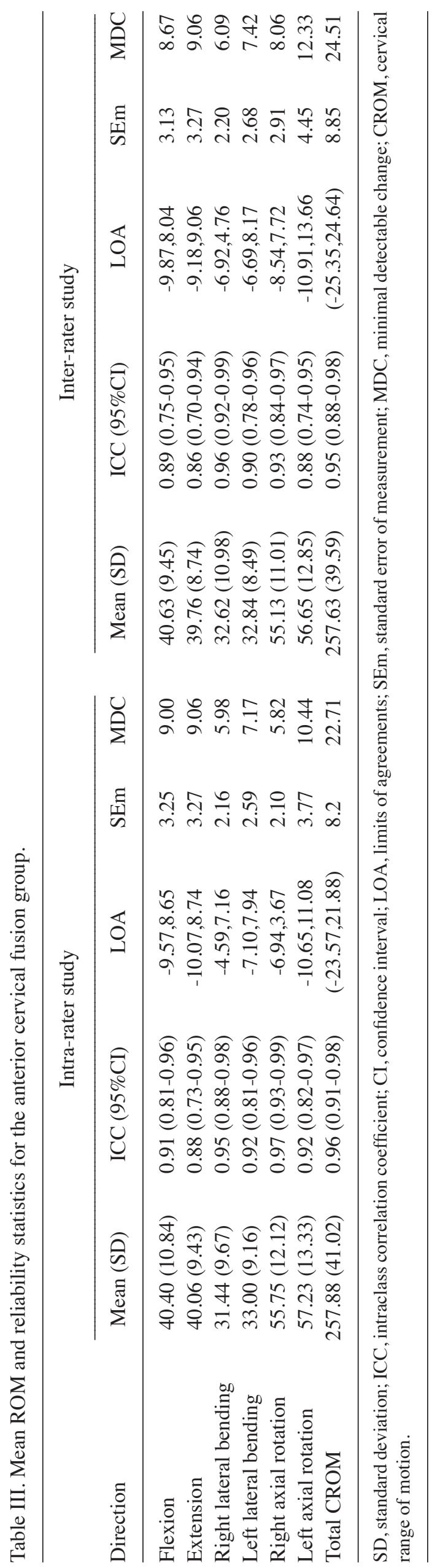

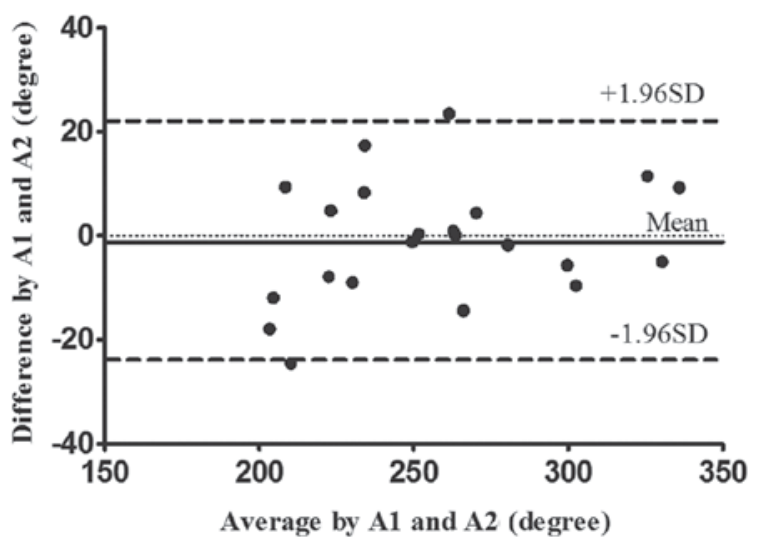

Figure 4. Intra-rater limits of agreements plots for total cervical range of motion of the anterior cervical fusion group. The solid line indicates the mean difference of the measurements, the upper and lower dashed lines indicate the $1.96 \mathrm{SD}$ above and below the mean difference of the measurements. SD, standard deviation; A1, first measurement by tester A; A2, second measurement by tester A (1 week after A1).

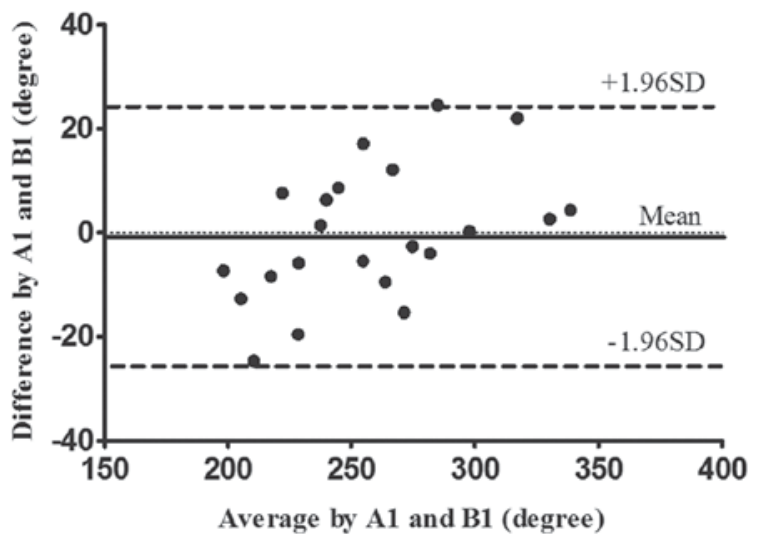

Figure 5. Inter-rater limits of agreements plots for total cervical range of motion of the anterior cervical fusion group. The solid line indicates the mean difference of the measurements, the upper and lower dashed lines indicate the $1.96 \mathrm{SD}$ above and below the mean difference of the measurements. $\mathrm{SD}$, standard deviation; $\mathrm{A} 1$, first measurement by tester $\mathrm{A} ; \mathrm{B} 1$, measurement by tester B (20 min after A1).

\section{Discussion}

Numerous instruments have been used for the measurement of CROM, but they may cause human error due to the inclusion of trunk motion or the requirement for a tester to read the scale (8-10). Therefore, a 'better' approach should be identified to measure cervical mobility in clinical and research applications. In addition, most studies are currently focused on measuring the ROM of healthy people or patients with neck pain $(7,17,25)$, while the reliability of the measurements among cervical spondylosis and cervical fusion patients has remained to be determined. The CODA motion analysis system has not been used for measuring CROM, but has been reported to reliably and accurately evaluate the ROM of other joints under different circumstances $(15,16)$. To the best of our knowledge, the present study was the first to apply this system for measuring cervical mobility and proved to be highly reliable in determining the CROM. Besides, the construct validity 
Table IV. Differences in CROM between cervical spondylosis and anterior cervical fusion group.

\begin{tabular}{lccc}
\hline Direction & Cervical spondylosis group & Anterior cervical fusion group & P-value \\
\hline Flexion & $47.4 \pm 9.3$ & $40.6 \pm 9.6$ & 0.005 \\
Extension & $48.0 \pm 12.6$ & $39.9 \pm 8.4$ & 0.015 \\
Right lateral bending & $39.1 \pm 12.7$ & $32.0 \pm 10.3$ & 0.041 \\
Left lateral bending & $41.5 \pm 13.9$ & $32.8 \pm 8.6$ & 0.015 \\
Right axial rotation & $65.4 \pm 13.4$ & $55.6 \pm 11.3$ & 0.010 \\
Left axial rotation & $62.7 \pm 13.8$ & $56.8 \pm 12.7$ & 0.120 \\
Total CROM & $305.1 \pm 51.1$ & $257.5 \pm 40.3$ & 0.012
\end{tabular}

CROM, cervical range of motion.

was verified because the system was sensitive enough to distinguish the CROM between cervical spondylosis and $\mathrm{ACF}$ group.

As an important indicator of cervical function, the three-dimensional CROM of cervical spondylosis patients has been assessed by various devices in previous studies. Dvir et al (26) utilized the Zebris system to assess the ROM of patients with cervical degenerative disease, the results were 82.5 for flexion-extension, 65.3 for lateral bending, and 103.9 for axial rotation. Yuan et al (4) used the CROM device to measure the ROM of patients with cervical myelopathy, and the results were 90.2 for flexion-extension, 83.2 for lateral bending and 114 for axial rotation. The CROM of ACF patients was also assessed using other methods. Cattrysse et al (27) used the electromagnetic tracking system to assess the ROM of patients who received ACF, and the results were 46.94-66.87 for lateral bending and 95.81-112.59 for axial rotation. Bell et al (28) used a virtual reality-assisted cervical motion tracking device (VR-assisted CMTD) to assess the ROM of patients who received ACDF, and the ROM was 67.2-89.7 for flexion-extension, 39.2-65.8 for lateral bending and 83.3-111.7 for axial rotation. In the present study, the CODA motion analysis system was utilized to measure the three-dimensional CROM of cervical spondylosis and ACF patients. Comparison of the present results with those of the previous studies indicated that in the cervical spondylosis group, the CODA motion analysis system was able to obtain a similar ROM to that determined by the CROM device and Zebris system, and in the ACF group, it determined a similar ROM to that obtained with an electromagnetic tracking system and VR-assisted CMTD. The similarity of the ROM assessed by various devices proved that the CODA motion analysis system is able to accurately measure the three-dimensional CROM.

Most previous studies only used ICC to assess the reliability of devices, which greatly reduced the credibility of their results $(25,29)$. The present study comprehensively evaluated the reliability of the CODA motion analysis system by calculating ICC, LOA, SEm and MDC. The intra- and inter-rater ICCs in the cervical spondylosis group were 0.87-0.95 and 0.84-0.95, respectively. The intra- and inter-rater ICCs in the cervical fusion group were 0.88-0.97 and 0.86-0.96, respectively. Therefore, the reliability of the CODA motion analysis system was sufficiently high to render it suitable for use in clinical and research settings for the measurement of CROM in all directions. Various studies have also assessed the reliability of other devices among individuals with cervical disorders. Williams et al (9) assessed the reliability of the CROM device among cervical whiplash patients and obtained ICC values of 0.98-0.99 for intra-rater reliability and $0.82-0.92$ for inter-rater reliability. Dvir et al (26) evaluated the reliability of the Zebris system for patients with degenerative changes, and the ICCs for intra-rater reliability were in the range of 0.80-0.89. Law and Chiu (25) assessed the reliability of the Electronic CROM Goniometer among patients with neck pain, and the ICCs for inter-rater reliability ranged from 0.81 to 0.92 . In comparison with that of the other devices, the reliability of the CODA motion analysis system was equal or superior.

In the present study, almost all ICCs of flexion and extension were $<0.90$, which was lower than that of the other four directions. During the measurements, the marker attached to the incisure jugular was probably obscured by the jaw during the flexion, while during the extension, the marker attached to the occipital protuberance was likely obscured by the wrinkle of the neck skin. This obscuring effect may have resulted in the lower ICCs of flexion and extension. Furthermore, the ICCs of almost all directions in the cervical spondylosis group (range, 0.84-0.95) were lower than those in the cervical fusion group (range, 0.86-0.97). One possible explanation is that the lower CROM in the cervical fusion group may reduce the obscuring of the markers, which is beneficial for achieving higher ICCs.

The calculation of the MDC value is essential in assessing the reliability of a novel device. In the cervical spondylosis group, MDCs were 8.23-12.69 for intra-rater reliability, and 8.67-11.97 for inter-rater reliability. In the ACF group, the MDCs were 5.82-10.44 for intra-rater reliability and 6.09-12.33 for inter-rater reliability. MDC values for CROM have also been reported in previous studies. Shahidi et al (30) used a gravity inclinometer to measure CROM and the MDC ranged from 9 to 21 regarding inter-rater reliability. The lower MDCs in the present study indicated that the CODA motion analysis system is sensitive in detecting small changes in three-dimensional CROM. Therefore, the system is adequate to be applied in clinical and research practices.

The assessment of construct validity was performed by comparing the three-dimensional CROM between cervical spondylosis and ACF groups. The results revealed that the CROM in the cervical spondylosis group was significantly higher than that in the ACF group in all directions except 
for left rotation, supporting the ability of the CODA motion analysis system to discriminate between cervical spondylosis and ACF patients. The lower CROM for the ACF group was in agreement with the results of studies (31-33). These studies often calculated the C2-7 ROM of flexion and extension by lateral radiographs. In a study by Lee et al (31), an increase in the C0-2 ROM was observed after ACF, although the difference was not significant. However, the results of three-dimensional studies were contrary to the above. The study by Bell et al (28) revealed that the postoperative CROM in the 1-or 2-level ACDF group was higher than the preoperative one. A study by Landers et al (34) revealed that the CROM increased significantly after ACDF regardless of the number of levels fused. The reason for the difference in results between these studies may be attributed to the compensation of the $\mathrm{C} 0-2 \mathrm{ROM}$, the options of anterior fusion methods and the number of fused levels. Due to the conflicting results regarding CROM after $\mathrm{ACF}$, the study of three-dimensional motion of the cervical spine is gaining importance. In the present study, although the left rotation ROM in the cervical spondylosis group was higher than that in the ACF group, the difference between the groups was not significant. One possible reason for the equal results regarding left rotation may be that the rotation of the cervical spine is mainly performed by the upper cervical spine, particularly $\mathrm{C} 1$ and $\mathrm{C} 2(35,36)$, while anterior fusion surgery is often performed between $\mathrm{C} 3$ and $\mathrm{C} 7$, and the ROM of rotation may thus be less influenced by the surgery.

The present study had two limitations. First, the markers are not stationary with respect to the underlying bone due to the soft tissue between the markers and the bone landmarks. Therefore, the system may not be suitable for measuring the ROM of obese patients, since the soft tissue may be thicker. Furthermore, the system is expensive, which may limit its application in clinical practice. However, taking into account its various uses, such as assessing gait posture (13), lower and upper extremity kinematics $(14,15)$ and lumbar spine ROM, the high price may not restrict its application in clinical practice.

In conclusion, the present study confirmed the high reliability of the CODA motion analysis system in the measurement of CROM among cervical spondylosis and ACF patients. The construct validity of the CODA motion analysis system was verified, as the system was sufficiently sensitive to distinguish between cervical spondylosis and ACF based on the CROM.

\section{References}

1. Takagi I, Eliyas JK and Stadlan N: Cervical spondylosis: An update on pathophysiology, clinical manifestation, and management strategies. Dis Mon 57: 583-591, 2011.

2. Nagamoto Y, Ishii T, Sakaura H, Iwasaki M, Moritomo H, Kashii M, Hattori T, Yoshikawa $\mathrm{H}$ and Sugamoto K: In vivo three-dimensional kinematics of the cervical spine during head rotation in patients with cervical spondylosis. Spine (Phila Pa 1976) 36: 778-783, 2011.

3. Miyazaki M, Hong SW, Yoon SH, Zou J, Tow B, Alanay A, Abitbol JJ and Wang JC: Kinematic analysis of the relationship between the grade of disc degeneration and motion unit of the cervical spine. Spine (Phila Pa 1976) 33: 187-193, 2008.

4. Yuan W, Zhu Y, Liu X, Zhu H, Zhou X, Zhou R, Cui C and Li J: Postoperative three-dimensional cervical range of motion and neurological outcomes in patients with cervical ossification of the posterior longitudinal ligament: Cervical laminoplasty versus laminectomy with fusion. Clin Neurol Neurosurg 134: 17-23, 2015.
5. Bogduk $\mathrm{N}$ and Mercer S: Biomechanics of the cervical spine I: Normal kinematics. Clin Biomech (Bristol, Avon) 15: 633-648, 2000.

6. Maksymowych WP, Mallon C, Richardson R, Conner-Spady B, Jauregui E, Chung C, Zappala L, Pile K and Russell AS: Development and validation of a simple tape-based measurement tool for recording cervical rotation in patients with ankylosing spondylitis: Comparison with a goniometer-based approach. J Rheumatol 33: 2242-2249, 2006.

7. Lachtman DS, Bartha DA, Beltran MM, Dominguez DN, Messerli AR, Miller SE, Davis AM and Nelson-Wong E: Rater reliability and concurrent validity of single and dual bubble inclinometry to assess cervical lateral flexion. J Manipulative Physiol Ther 38: 572-580, 2015.

8. Dvorák J, Panjabi MM, Grob D, Novotny JE and Antinnes JA Clinical validation of functional flexion/extension radiographs of the cervical spine. Spine (Phila Pa 1976) 18: 120-127, 1993.

9. Williams MA, Williamson E, Gates S and Cooke MW: Reproducibility of the cervical range of motion (CROM) device for individuals with sub-acute whiplash associated disorders. Eur Spine J 21: 872-878, 2012.

10. Jordan K, Dziedzic K, Jones PW, Ong BN and Dawes PT: The reliability of the three-dimensional FASTRAK measurement system in measuring cervical spine and shoulder range of motion in healthy subjects. Rheumatology (Oxford) 39: 382-388, 2000.

11. Cagnie B, Cools A, De Loose V, Cambier D and Danneels L: Reliability and normative database of the Zebris cervical range-of-motion system in healthy controls with preliminary validation in a group of patients with neck pain. J Manipulative Physiol Ther 30: 450-455, 2007.

12. Bulgheroni MV, Antonaci F, Ghirmai S, Sandrini G, Nappi G and Pedotti A: A 3D kinematic method for evaluating voluntary movements of the cervical spine in humans. Funct Neurol 13: 239-245, 1998.

13. Exell TA, Gittoes MJ, Irwin G and Kerwin DG: Gait asymmetry: Composite scores for mechanical analyses of sprint running. J Biomech 45: 1108-1111, 2012.

14. Delahunt E, Cusack K, Wilson L and Doherty C: Joint mobilization acutely improves landing kinematics in chronic ankle instability. Med Sci Sports Exerc 45: 514-519, 2013.

15. Levanon Y, Gefen A, Lerman Y, Givon U and Ratzon NZ: Validity and reliability of upper extremity three-dimensional kinematics during a typing task. Gait Posture 32: 469-474, 2010.

16. O'Sullivan K, Clifford A and Hughes L: The reliability of the CODA motion analysis system for lumbar spine analysis: A pilot study. Physiotherapy Pract Res 31: 16-22, 2010.

17. Williams MA, McCarthy CJ, Chorti A, Cooke MW and Gates S: A systematic review of reliability and validity studies of methods for measuring active and passive cervical range of motion. J Manipulative Physiol Ther 33: 138-155, 2010.

18. Price DD, McGrath PA, Rafii A and Buckingham B: The validation of visual analogue scales as ratio scale measures for chronic and experimental pain. Pain 17: 45-56, 1983.

19. Fukui M, Chiba K, Kawakami M, Kikuchi S, Konno S, Miyamoto M, Seichi A, Shimamura T, Shirado O, Taguchi T, et al: Japanese orthopaedic association cervical myelopathy evaluation questionnaire (JOACMEQ): Part 2. Endorsement of the alternative item. J Orthop Sci 12: 241-248, 2007.

20. Stratford PW and Goldsmith $\mathrm{CH}$ : Use of the standard error as a reliability index of interest: An applied example using elbow flexor strength data. Phys Ther 77: 745-750, 1997.

21. Beaton DE, Bombardier C, Katz JN and Wright JG: A taxonomy for responsiveness. J Clin Epidemiol 54: 1204-1217, 2001.

22. Bland JM and Altman DG: Agreed statistics: Measurement method comparison. Anesthesiology 116: 182-185, 2012.

23. Shrout PE: Measurement reliability and agreement in psychiatry. Stat Methods Med Res 7: 301-317, 1998

24. Nussbaumer S, Leunig M, Glatthorn JF, Stauffacher S, Gerber H and Maffiuletti NA: Validity and test-retest reliability of manual goniometers for measuring passive hip range of motion in femoroacetabular impingement patients. BMC Musculoskelet Disord 11: 194, 2010.

25. Law EY and Chiu TT: Measurement of cervical range of motion (CROM) by electronic CROM goniometer: A test of reliability and validity. J Back Musculoskelet Rehabil 26: 141-148, 2013

26. Dvir Z, Gal-Eshel N, Shamir B, Prushansky T, Pevzner E and Peretz C: Cervical motion in patients with chronic disorders of the cervical spine: A reproducibility study. Spine (Phila $\mathrm{Pa}$ 1976) 31: E394-E399, 2006. 
27. Cattrysse E, Moens M, Schaillée E, D'Haens J and Van Roy P Changed cervical kinematics after fusion surgery. Eur Spine J 21: 1353-1359, 2012.

28. Bell KM, Bechara BP, Hartman RA, Shively C, Frazier EC, Lee JY, Kang JD and Donaldson WF: Influence of number of operated levels and postoperative time on active range of motion following anterior cervical decompression and fusion procedures. Spine (Phila Pa 1976) 36: 263-268, 2011.

29. Quek J, Brauer SG, Treleaven J, Pua YH, Mentiplay B and Clark RA: Validity and intra-rater reliability of an android phone application to measure cervical range-of-motion. J Neuroeng Rehabil 11: 65, 2014.

30. Shahidi B, Johnson CL, Curran-Everett D and Maluf KS: Reliability and group differences in quantitative cervicothoracic measures among individuals with and without chronic neck pain BMC Musculoskelet Disord 13: 215, 2012.

31. Lee SH, Lee JC, Tauchi R and Daniel Riew K: Influence of the number of cervical fusion levels on cervical spine motion and health-related quality of life. Spine (Phila Pa 1976) 41: E474-E480, 2016.
32. Chien A, Lai DM, Wang SF, Cheng CH, Hsu WL and Wang JL: Differential segmental motion contribution of single- and two-level anterior cervical discectomy and fusion. Eur Spine J 24: 2857-2865, 2015.

33. Mao N, Wu J, Zhang Y, Gu X, Wu Y, Lu C, Ding M, Lv R, Li M and Shi Z: A comparison of anterior cervical corpectomy and fusion combined with artificial disc replacement and cage fusion in patients with multilevel cervical spondylotic myelopathy. Spine (Phila Pa 1976) 40: 1277-1283, 2015.

34. Landers MR, Addis KA, Longhurst JK, Vom Steeg BL, Puentedura EJ and Daubs MD: Anterior cervical decompression and fusion on neck range of motion, pain and function: A prospective analysis. Spine J 13: 1650-1658, 2013.

35. Takasaki H, Hall T, Oshiro S, Kaneko S, Ikemoto Y and Jull G: Normal kinematics of the upper cervical spine during the Flexion-Rotation Test-In vivo measurements using magnetic resonance imaging. Man Ther 16: 167-171, 2011.

36. Rudolfsson T, Björklund $\mathrm{M}$ and Djupsjöbacka M: Range of motion in the upper and lower cervical spine in people with chronic neck pain. Man Ther 17: 53-59, 2012. 